\title{
Extremely heart-throb measurement based on laser self-mixing interferometer
}

\author{
Chao Wang ${ }^{1}$, jingyu Yang ${ }^{2}$, Zhiyuan $\mathrm{Li}^{3}$ \\ 713th RESEARCH INSTITUTE OF CHINA SHIPBUILDING INDUSTRY \\ 713th RESEARCH INSTITUTE OF CHINA SHIPBUILDING INDUSTRY \\ CORPORATION, Zhengzhou, China \\ CORPORATION, Zhengzhou, China \\ 854909839@qq.com,854909839@qq.com
}

Keywords: heart-throb; laser self-mixing; fringe counting algorithm

\begin{abstract}
In this paper, A heart-throb acquisition technique based on laser self-mixing is proposed. In view of the characteristics of extremely weak vibration of human heart-throb, a multiple reflection method is proposed. The objective of the external cavity is to improve the measurement accuracy by $\lambda / 5$. Fringe counting algorithm, A periodic heart-throb is successfully reconstructed. The measurement results show that the root mean square error (RMSE) the reconstructed heartthrob was $0.813 \mu \mathrm{m}$ at $9 \mathrm{kHz}$ sampling rate. In addition, correlation coefficients. And heartbeat were analyzed by this method and conventional photoelectric volume meter (PPG) measurements.
\end{abstract}

\section{Introduction}

With the development of science and technology and the needs of people's daily life production .In order to improve the accuracy of the micro displacement measurement of heart-throb, more and more attention has been paid to the accuracy of the micro displacement measurement.Micro displacement measurement of pulse by photoelectric encoder and digital encoder.It has been widely used in quantity.

Photoelectric encoder is divided into absolute encoder and its encoder according to its counting principle.There are two kinds of incremental encoders, and the absolute encoders can be based on the corresponding corners.The corresponding code is given according to the change; the increment encoder calculates the code according to the calculation.The pulse increment generated by the disk rotation is modified according to a reference number.Angular displacement is obtained. The encoder used in the encoder technology is made up of the structure.It can be divided into contact encoder and optical encoder. Contact knitting.According to the principle, the encoder is a kind of absolute encoder, which consists of a coded disk,Brush and circuit composition. The disadvantage is that the contact between the encoder and the brush will produce.Life friction shortens service life. Incorrect connection of brush and code track.Touch also produces fuzzy output, which may give erroneous results causing errors Poor. There is also an absolute encoder that is optical encoder.It's the basis devices made by optical and optoelectronic principles. Optical encoder consists of light source,The encoder, the optical system and the corresponding circuit are composed of four parts. The device is a kind of mechanical displacement on the output shaft by photoelectric conversion.This is the most widely used Multiple sensors.

\section{Algorithm analysis}

The origin of the laser self mixing interference theory to found the laser feedback light can be scattered into the laser cavity. And this part of the reflected light can severely affect the performance of the laser, people how to eliminate the influence of light reflection in the study also found the influence on the laser power law, and gradually formed the theory of laser self mixing interference. The laser self mixing interferometer has the advantages of simple structure, high sensitivity, low cost, 
wide application range and widely used. Since the position change of mixed interference theory of reflective object in laser cavity and corresponding intensity fluctuation, a fringe fluctuation corresponding to the external cavity reflecting half a wavelength position change, by counting the number of cycles can be measured on the displacement. When the feedback light reaches a certain level, the interference fringes for sinusoidal waveform tilt, consistent tilt direction and displacement direction, due to the interference signals can reflect the displacement of the object, which can distinguish the direction of movement of the object. Based on an analytical steady-state solution, the emitted power $\mathrm{P}$ is usually expressed as:

$$
\mathrm{P}(\phi)=\mathrm{P}_{0}[1+\mathrm{m} \mathrm{F}(\phi)]
$$

which is amplitude modulated by a periodic interferome-tric function $\mathrm{F}(\phi)$, whose period is with phase shift of $2 \pi$. In Eq.(1), $P_{0}$ is the laser power without optical feedback and $m$ is the modulation index; $\phi$ is the optical phase shift of the external path with feedback, given by $\phi$ $=2 \mathrm{~kL}=4 \pi \mathrm{L} / \lambda$ with $\mathrm{k}$ as the wave vector, $\lambda$ as the wave-length, and $\mathrm{L}$ as the variation of optical distance from the laser diode (LD) to the reflector. It is recognized that the phase difference is caused by the external optical path difference.

The origin of the laser self mixing interference theory to found the laser feedback light can be scattered into the laser cavity. And this part of the reflected light can severely affect the performance of the laser, people how to eliminate the influence of light reflection in the study also found the influence on the laser power law, and gradually formed the theory of laser self mixing interference. The laser self mixing interferometer has the advantages of simple structure, high sensitivity, low cost, wide application range and widely used. Since the position change of mixed interference theory of reflective object in laser cavity and corresponding intensity fluctuation, a fringe fluctuation corresponding to the external cavity reflecting half a wavelength position change, by counting the number of cycles can be measured on the displacement. When the feedback light reaches a certain level, the interference fringes for sinusoidal waveform tilt, consistent tilt direction and displacement direction, due to the interference signals can reflect the displacement of the object, which can distinguish the direction of movement of the object.

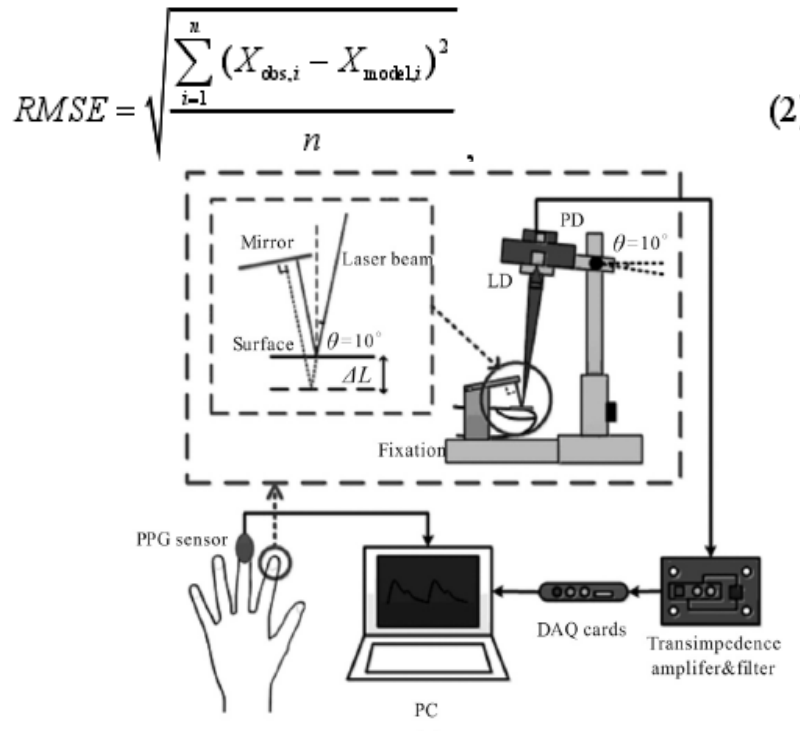

Fig.1 (a) Schematic diagram of the proposed experimental setup; (b) Photograph of the experimental setup: (1) Laser diode, (2) External mirror, (3) Fixation, (4)Transimpedance

In Xobs observations, the $\mathrm{X}$ model has some reference value, while Xcorr is calculated using the algorithm in the literature[17]:

$$
X_{\text {car }}(n)=\frac{R_{x y}(n)}{\left|R_{x x}(0) R_{y y}(0)\right|^{1 / 2}},
$$

The estimation of the cross covariance of $R_{x y}(n)$ is defined as 


$$
R_{k y}(n)= \begin{cases}\frac{1}{N} \sum_{k=1}^{N-n-1} x(k) y(k+n), & n=0,1,2, \ldots, N \\ \frac{1}{N} \sum_{k=0}^{N+n-1} x(k-n) y(k), & n=0,-1,-2, \ldots, N,\end{cases}
$$

and

$$
\begin{aligned}
& R_{x x}(0)=\frac{1}{N} \sum_{k=0}^{N-1}[x(k)]^{2}, \\
& R_{y}(0)=\frac{1}{N} \sum_{k=0}^{N-1}[y(k)]^{2},
\end{aligned}
$$

Among them, $x(k)$ is the data sample of heart-throb obtained by PPG and Y $(k)$, which is the data obtained by SMI to reconstruct heart-throb. $N$ is the length of two sequences of $x(k)$ and $y(k)$.

Self mixing interference system device are shown in Fig.2. ${ }^{[18,19]}$. Fig.2(a) shows Schematic diagram of the proposed experimental setup; Photograph of the experimental setup as shown in Fig.2(b). The $\mathrm{L}$ output beam of the laser is returned to the laser cavity by the T part of the external reflection and mixed with the intracavity beam. The light intensity of the laser is detected by the detector $\mathrm{D}$ [3]. When the external reflection object changes the position of the half wave length, the laser will produce an output power stripe change, and when the optical feedback reaches a certain level, the power stripe is quasi sawtooth wave shape. The dip direction of the sawtooth wave is related to the moving direction of the object. This feature can be used to determine the direction directly.

The $\mathrm{L}$ output beam of the laser is returned to the laser cavity by the $\mathrm{T}$ part of the external reflection and mixed with the intracavity beam. The light intensity of the laser is detected by the detector $\mathrm{D}$ [4]. When the external reflection object changes the position of the half wave length, the laser will produce an output power stripe change, and when the optical feedback reaches a certain level, the power stripe is quasi sawtooth wave shape. The dip direction of the sawtooth wave is related to the moving direction of the object. This feature can be used to determine the direction directly. Comparing Fig.2(b) with Fig.2(c), the fringe numbers of the arterial pulse wave are 56 and 110 in the premier $0.1 \mathrm{~s}$.

The theoretical results show that the self mixing interference and cavity position change of reflective object corresponding to the laser intensity fluctuation, a fringe fluctuation corresponding to the external cavity reflecting half a wavelength position change, by counting the number of cycles of displacement measurement.

When the feedback light reaches a certain level, the interference fringe is tilted like sinusoidal waveform, and the tilt direction is consistent with the displacement direction of the object. Because the interference signal can reflect the direction of the object displacement, the direction of the object can be distinguished. In addition, the displacement vector can be measured directly by the displacement interference signal when the system is in bistablestate.
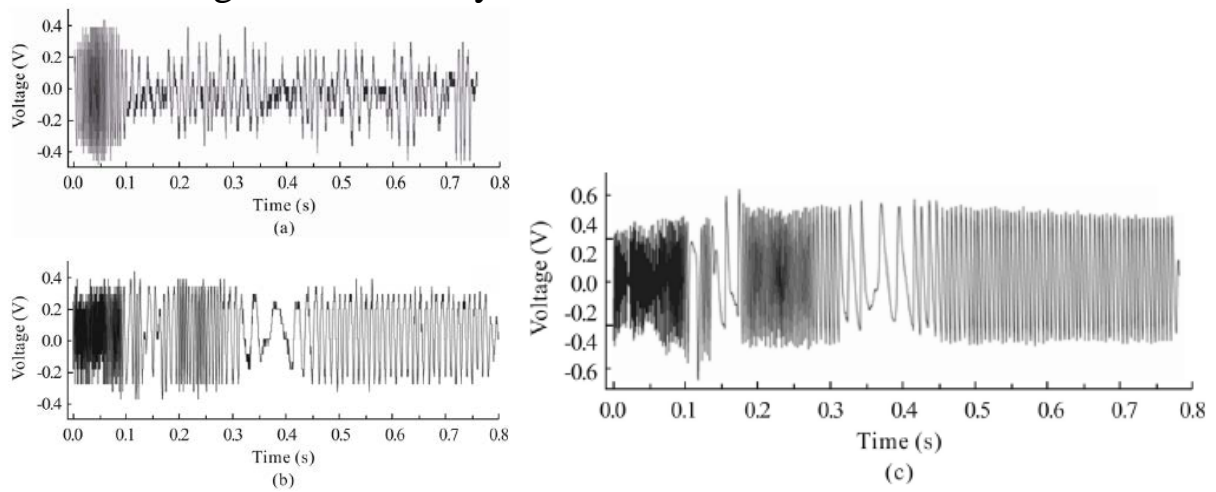

Fig.2 Measured SMI signals in the case of (a) naked fingernail, (b) a thin mirror on the fingernail and (c) an external reflecting mirror 
As three curves shown in Fig.3, the reconstructed signals with $\lambda / 2$ resolution and $\lambda / 4$ resolution are well superposed. The laser diode model used in this experiment is QL65D5SA. Because the wavelength of laser is related to its working temperature to a certain extent, it is usually QL65D5SA.The visible light output wavelength is $25, \mathrm{P}=650 \mathrm{~nm}$, and the optical power output is $5 \mathrm{~mW}$, rated voltage is $2.2 \mathrm{~V}$, rated current is $28 \mathrm{~mA}$. So here the driver chip for laser diode is WLD3343, WLD3344.2.2A laser diode driver, driving circuit as shown in Figure 3, the VSET pin is calculated according to the set to $2 \mathrm{~V}$, the remaining parts of sliding rheostat resistance can be calculated according to the chip data obtained.

As shown in Figure 4, with the current flowing through the rotation of the Pulse to the right when the laser diode reflectors by AD620 amplification, the self mixing interference fringes as saw tooth wave shape, sawtooth wave shape is obtained by the semiconductor laser in the bistable case secondary feedback level.

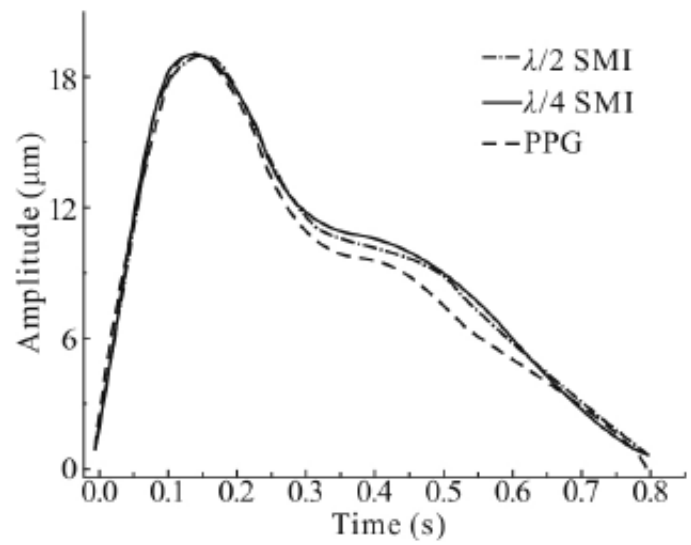

Fig.3 Pulse signals of reconstruction

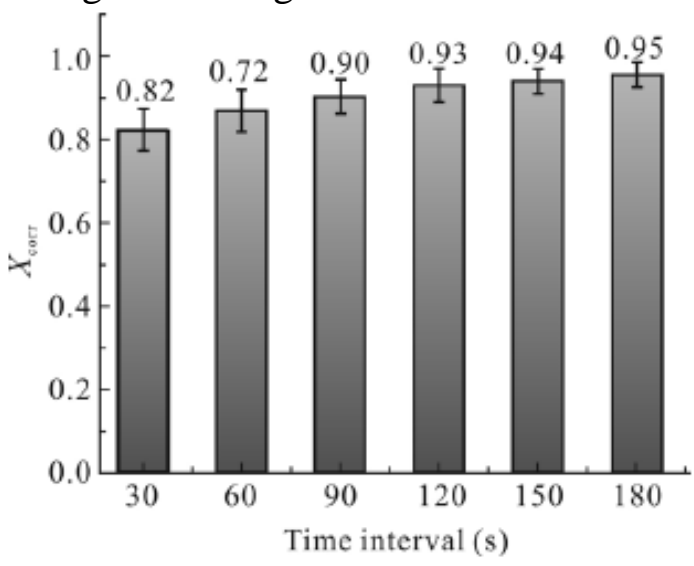

Fig.4 Correlation coefficients $\left(\mathrm{X}_{\text {corr }}\right)$ and standard deviations under different measurement periods of time

Fig.5(a) shows, when the Heart beat with reflective stickers rotate, the laser self mixing interference produces interference fringes, and the saw tooth wave inclination direction corresponds to the wheel rotation direction. The reflected light scatters to the laser due to the distance of the reflected object moving half a wavelength.Power changes caused by mixing the cavity and mixing with laser. From Fig.5(b), The corresponding external object moves about half a wavelength, and the experimental waveform produces a stripe change. According to this, the displacement of external object can be measured or measured, and the resolution is half wavelength with laser self mixing. Due to the laser diode wavelength lambda, $\mathrm{P}=650 \mathrm{~nm}$, the resolution of the system is the rotation arc length corresponding to a self mixing interference fringe.

In this paper, the principle of photoelectric encoder is introduced in this paper. The accuracy of the self mixing laser displacement measurement is compared with the calculation accuracy of the universal 1000 wire encoder. The $n=1000$, the radian corresponding to a pulse $=p i / 500$, the distance traveled by the wheel is equal to the beta $=\mathrm{R} / 500$, so the resolution of the photoelectric 
encoder speed measurement system is at this time.

If the value of $\mathrm{R}$ is $0.01 \mathrm{M}$, the corresponding distance of each pulse generated by the encoder is about $0.0628 \mathrm{~mm}$.

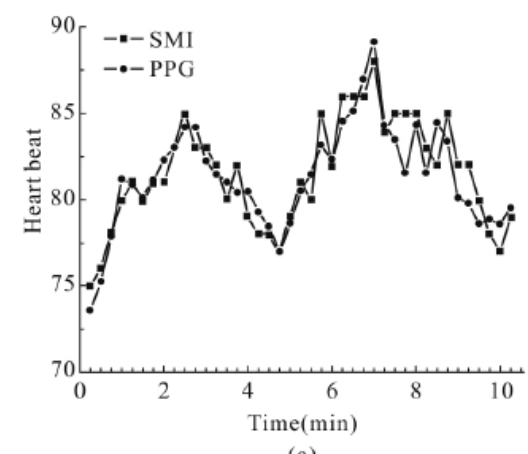

(a)

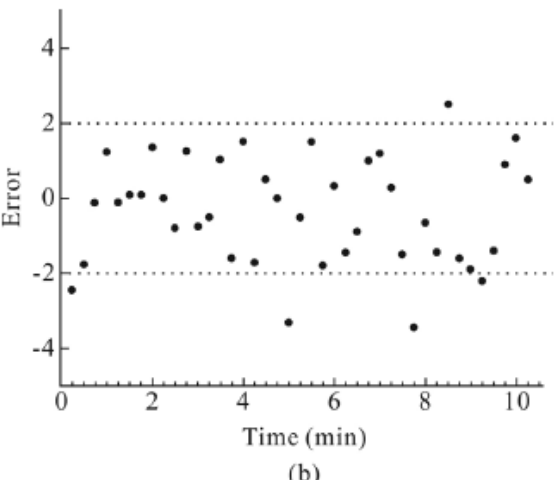

(b)

Fig.5 (a) Heart beats with different methods; (b) Errors of these two methods

\section{Conclusion}

Laser self mixing interference measurement technology is a new interferometer counting. It has simple structure, high resolution and high performance price ratio. This experiment adopts the self mixing interference fringe counting method based on micro displacement measurement, the experiments show that using the self mixing laser displacement measurement resolution of half a wavelength of the laser, the experiment due to the wavelength, $\mathrm{P}=650 \mathrm{~nm}$, the laser self mixing experiment to achieve a resolution of about $10 \mathrm{~m}$. Compared with the traditional 1000 wire photoelectric encoder, the resolution $0.0628 \mathrm{~mm}$ can be greatly improved, and it can be adapted to the measurement of micro displacement with high precision in various situations. There are many defects and deficiencies in this experiment: using laser self mixing interference counting to measure the displacement, we must first ensure that the wavelength of the laser can be stable, so that the drive circuit can provide a stable voltage and current to the laser diode. This requires higher laser operating temperature.

It is necessary to provide a stable self feedback temperature control circuit for the laser. At this point, there is still a great room for improvement in this experiment.

\section{References}

[1] Hongtao LU,Shihai SHAO,Kai DENG,Youxi TANG. Self-mixed self-interference analog cancellation in full-duplex communications[J]. Science China(Information Sciences),2016,59(04):31-44.

[2] Wei Xia,Ming Wang,Wenhua Guo Department of Physics,Nanjing Normal University,Nanjing 210097,P.R.China. A Laser Self-mixing Interference Vibrometer Based On Current Modulation and DSP Demodulation[A]:2010:6.

[3] Jiang Chunlei. EMD filter algorithm for self-mixing interference displacement sensor. IEEE Beijing Section、Chinese Institute of Electronics.Proceedings of 2015 12th IEEE International Conference on Electronic Measurement \& Instruments[C].IEEE Beijing Section、Chinese Institute of Electronics:2015:5.

[4] 王秀琳,王路,陈敏亮,黄文财. Micro-vibration parameters fast demodulation algorithm and experiment of self-mixing interference[J]. Optoelectronics Letters,2014,10(04):304-307.

[5] Hui Hao,Ming Wang,Dongmei Guo,Wei Xia. Jiangsu Key Lab on Opto-Electronic Technology, Proceedings of Conference 7844 Semiconductor Lasers ,Solid State Lighting \& Applications[C].中 国光学学会 (COS) 、国际光学工程学会 (SPIE) :2010:7. 
[6] Wei Z, Huang W, Zhang J, et al. Obtaining Scalable Fringe Precision in Self-Mixing Interference Using an Even-Power Fast Algorithm[J]. IEEE Photonics Journal, 2017, 9(4):1-11.

[7] Chen M, Zhang Y, Chen C, et al. Damping Microvibration Measurement Using Laser Diode Self-Mixing Interference[J]. IEEE Photonics Journal, 2013, 6(3):1-8.

[8] Jiang C, Li C. EMD filter algorithm for self-mixing interference displacement sensor[C]// IEEE International Conference on Electronic Measurement \& Instruments. IEEE, 2016:1603-1607.

[9] Yin S, Huang C. Research on micro-vibration measurement technique based on laser self-mixing interference[C]// International Conference on Measurement, Information and Control. IEEE, 2014:85-88.

[10] Wang L, Luo X, Wang X, et al. Obtaining High Fringe Precision in Self-Mixing Interference Using a Simple External Reflecting Mirror[J]. IEEE Photonics Journal, 2013, 5(3):207-217.

[11] Lei M, Zhang M, Yang Z, et al. Study on the self-mixing interference in a composite fourmirror cavity laser[C]// Lasers \& Electro Optics \& The Pacific Rim Conference on Lasers and Electro-Optics, 2009. CLEO/PACIFIC RIM '09. Conference on. IEEE, 2009:1-2.

[12] Choi M, Choi S. Performance analysis on the self-mixed interference cancellation in direct conversion receivers[J]. IEEE Transactions on Consumer Electronics, 2013, 59(2):310-315.

[13] Choi M, Choi S. Self-mixed interference cancellation method in direct conversion receivers[C]// IEEE International Conference on Consumer Electronics. IEEE, 2013:409-410.

[14] Gambini S, De Nardis L, Alon E, et al. Interference robust self-mixing UWB systems using phase-domain spreading[C]// IEEE International Conference on Ultra-Wideband. IEEE, 2011:44-48.

[15] Hao H, Wang M, Xia W, et al. Phase modulated self-mixing interferometer of an erbium-doped fiber laser[J]. Optics \& Laser Technology, 2013, 51(10):55-61.

[16] Lu H, Wang M, Dai X, et al. All-Fiber Self-Mixing Interferometer Based on DFB Laser and Phase Modulating Technique[J]. IEEE Photonics Technology Letters, 2011, 23(4):221-223.

[17] Zhang Y, Zheng M, Wei H, et al. Displacement Estimation Based on Phase Unwrapping Technique in Optical Self-mixing System[C]// First International Conference on Instrumentation, Measurement, Computer, Communication and Control. IEEE Computer Society, 2011:160-163.

[18] Larsson D, Greve A, Hvam J M, et al. Self-mixing interferometry in VCSELs for nanomechanical cantilever sensing[C]// Conference on Lasers and Electro-Optics and 2009 Conference on Quantum Electronics and Laser Science Conference. IEEE, 2009:1-2.

[19] Suleiman M, Han C S, Bosch T. Interrogation of Fiber Bragg Grating Dynamic Strain Sensors by Self-Mixing Interferometry[J]. IEEE Sensors Journal, 2008, 8(7):1317-1323.

[20] Larsson D, Yvind K, Hvam J M. Optimization of self-mixing modulation in VCSELs for sensing applications[C]// European Conference on Lasers and Electro-Optics 2009 and the European Quantum Electronics Conference. Cleo Europe - Eqec. IEEE, 2009:1-1. 